\title{
Routine hysterectomy should not be performed after radiotherapy end in women with endocervical adenocarcinoma
}

\author{
E.C.A. SOUZA, D.Z. SANTOS, N.G. SILVA, L.F. SALLUM, D.B. VALE, J.F. BRAGANCA, J. TEIXEIRA
}

Department of Gynecology and Obstetrics, Faculty of Medical Sciences

University of Campinas, Campinas (SP), Brazil

\section{AIMS}

Cervical cancer has an increasing proportion of the histological type adenocarcinoma (AC) in the last decades. Chemoradiation is the choice for inoperable cases, the same for squamous cell carcinomas histology. Considering the particularities of $A C$, there may be indication for hysterectomy after radiotherapy, but there is little information on the actual benefit. The aim of this study was evaluating hysterectomy post-radiotherapy in women with endocervical adenocarcinoma compared to those undergoing exclusive radiotherapy.

\section{METHODS}

There were selected 80 cases of women with endocervical adenocarcinomas FIGO IB to IIIB treated with radiotherapy and that had complete response: 39 received a post-radiation hysterectomy and 41 only follow-up, from 1988-2015 cared at University Hospital (Unicamp, Campinas-SP, Brazil). Diseasefree interval (DFI) and overall survival (OS) were analysed using Kaplan-Meier curves and log-rank test. This study was previously approved by local Ethics Committee.

\section{RESULTS}

There was no difference in OS between the groups $(p=0.579)$ or in the DFI $(p=0.963)$ [Figure 1]. Patients submitted to hysterectomy exhibited residual disease in uteri specimen in 56\% (22/39). It was observed that the DFI in patients with residual disease was lower, however without statistical significance $(p=0.072)$ [Figure 2]. Recurrences were observed in $9 / 39$ patients of the hysterectomy group and 10/41 patients of the isolated radiotherapy group. Residual disease in the hysterectomy group was associated with a higher rate of recurrence (local plus distant metastasis), 31\% (8/22 with residual disease) vs. $6 \%(1 / 17$, without residual disease) $(\mathrm{p}=0.028)$.
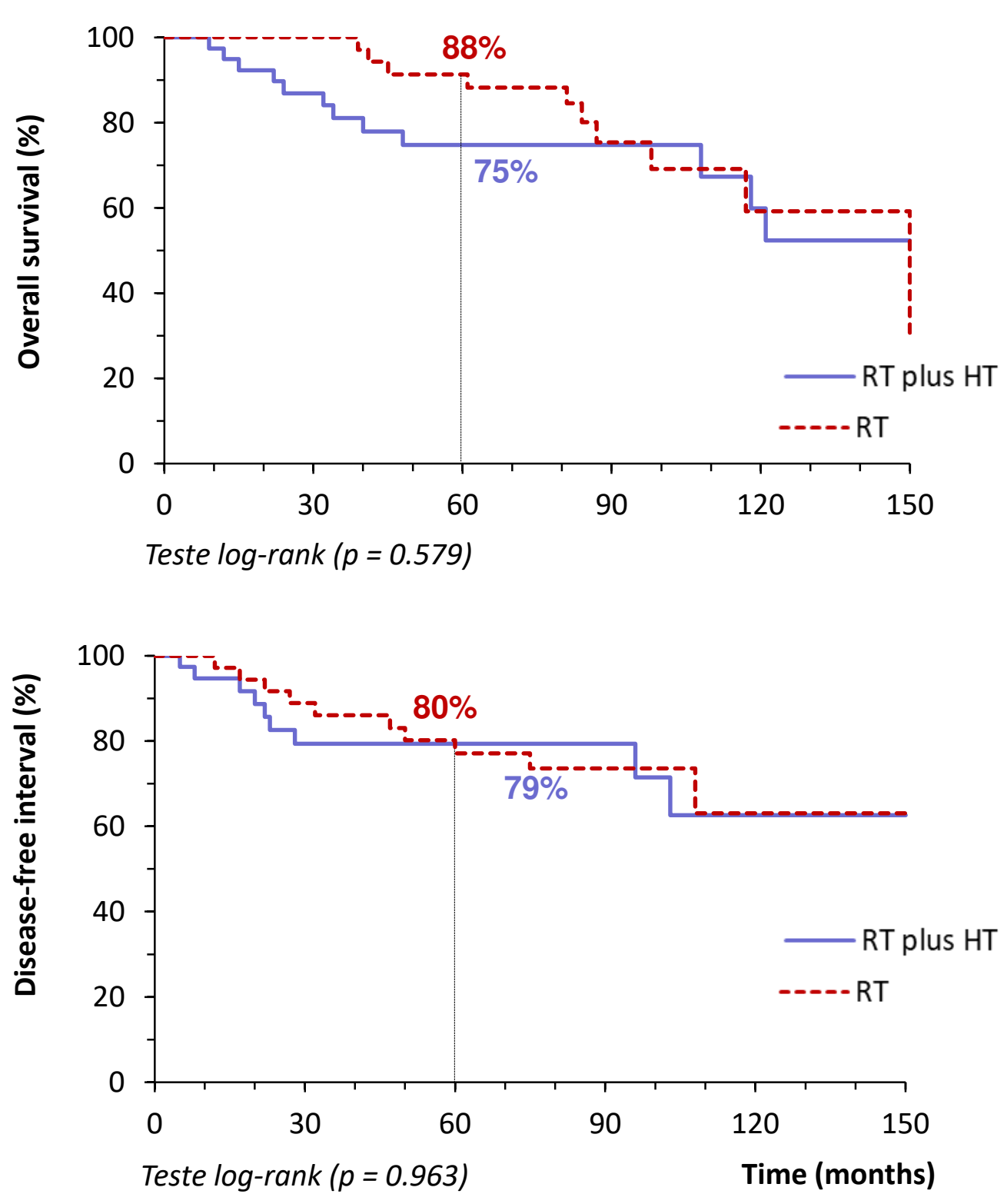

Figure 1: overall survival and disease-free interval of women with cervical adenocarcinoma treated with post-radiotherapy hysterectomy (RT plus HT $=39)$ or not $(R T=41)$.
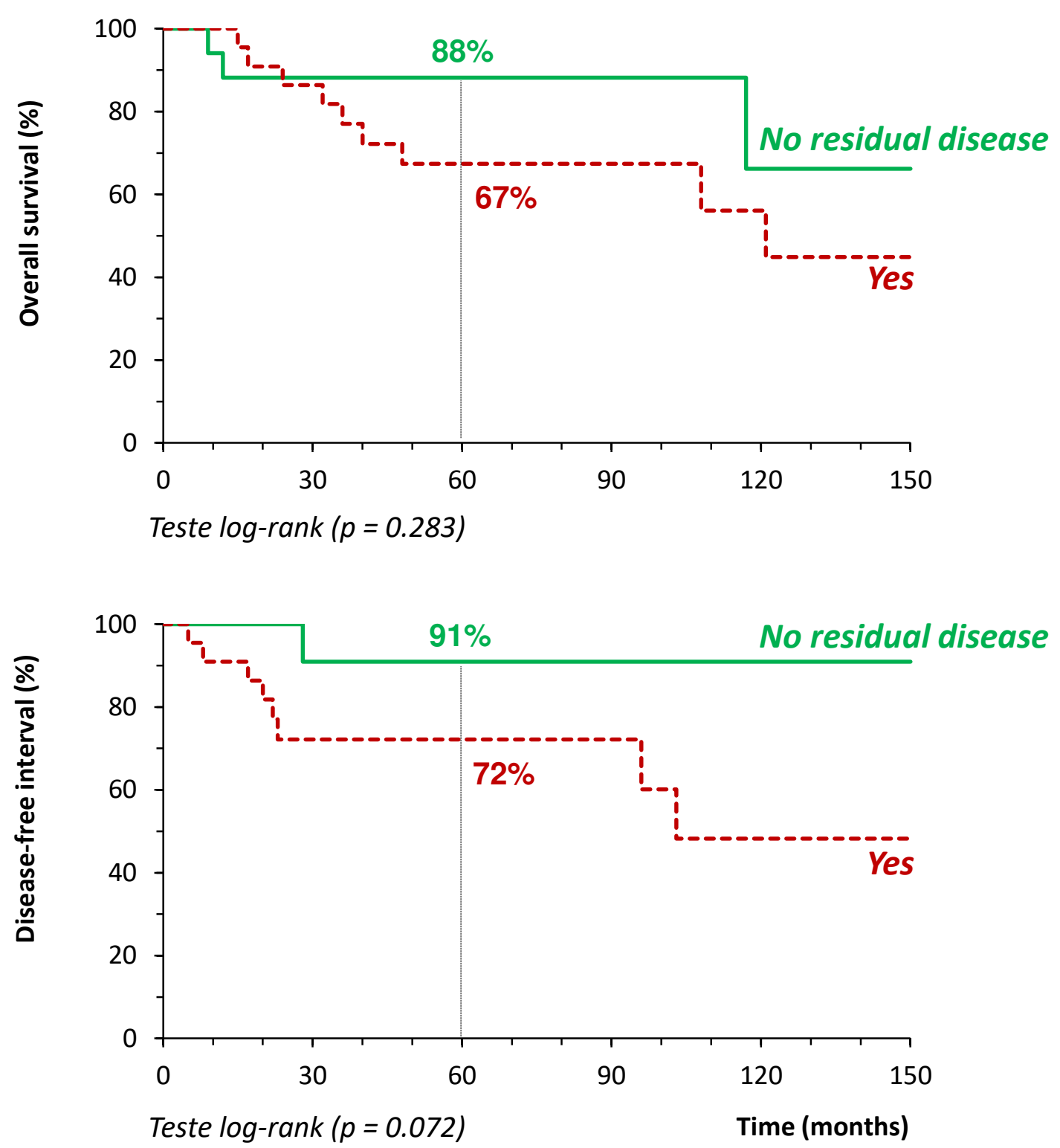

Figure 2: overall survival and disease-free interval according to presence of residual disease in specimens of postradiotherapy hysterectomy (No=17; Yes=22).

\section{CONCLUSIONS}

Post-radiotherapy hysterectomy for endocervical adenocarcinoma had no benefit in recurrence rates, disease-free interval, and overall survival, although residual disease in the hysterectomy specimen was associated with more recurrences. These findings do not support the routine hysterectomy in women previously irradiated in cervical adenocarcinoma. 